\title{
A FUZZY FRONT-END PRODUCT DEVELOPMENT FRAMEWORK FOR START-UPS
}

\author{
Martins Pacheco, Nuno Miguel; \\ Vazhapilli Sureshbabu, Anand; \\ Nürnberger, Masaru Charles; \\ Durán Noy, Laura Isabel; \\ Zimmermann, Markus \\ Technical University of Munich
}

\begin{abstract}
Start-ups tend to form with a central idea that differentiates them from their competitors in the market. It is crucial for them to efficiently transform the idea into a marketable product. Prototyping helps to iteratively achieve a minimum viable product and plays a crucial role by enabling teams to test their ideas with limited resources early on. However, the prototyping process may have wrong focus leading to a suboptimal allocation of resources. Previously, we proposed role-based prototyping for fuzzy front-end development in small teams. It supports (1) resource allocation, (2) the definition of responsibilities, and (3) structuring the development process with milestones. In recent research this was a promising yet incomplete approach. We extend the previous work by refining the prototyping process by adding a prototyping matrix with two dimensions (purpose and lens), a prototyping cycle (plan, execute, test, reflect, assimilate), and a modified Kanban board (Protoban) for planning, managing, and reflecting cycles. This process, named PETRA was tested with a start-up developing an autonomous trash picking robot. The extended approach supported the team significantly in providing a clear idea of what to do at what time.
\end{abstract}

Keywords: Early design phases, Collaborative design, Project management, New product development, User centred design

\section{Contact:}

Martins Pacheco, Nuno Miguel

Technical University of Munich

Product Development and Lightweight Design

Germany

martins.pacheco@tum.de 


\section{INTRODUCTION}

Start-ups tend to form around an idea and a unique selling proposition (USP) that differentiates them from their competitors in the market. These teams often have little to no experience on how to proceed with holistically developing a product. This is the case for many first-time entrepreneurs as they lack experience in product development processes. The development of new products is a complex, dynamic, iterative, and unique process. It often involves multidisciplinary teams with different perspectives to the development of new products (Karniel and Reich, 2011; Trott, 2016). Research has shown that teams with multiple disciplines accomplish a higher success rate in innovation development than teams of a single discipline (Crawford, 2008; Von Thienen et al., 2011).

The primary focus of our research is on new product development (NPD). NPD is characterized as the process of commercialization of a new product by transforming business opportunities into tangible products (Kahn et al., 2005; Trott, 2016). NPD is an integral part of the product innovation process and starts with recognizing the potential of an idea that leads to the product development (Florén et al., 2018; Trott, 2016). This research focusses on fuzzy front-end (FFE), new product development and commercialization when referring to NPD processes (Koen et al., 2002). In addition, this research refers to FFE as the messy, uncertain, chaotic, and early development phase (Gassmann and Schweitzer, 2014) of NPD. In this phase, main activities include customer needs identification, idea generation, idea evaluation, concept development, and concept evaluation (Gassmann and Schweitzer, 2014). The FFE ends when a go/no-go decision is taken based on available information, subsequently launching a formal new product development process with well-defined specifications for prototyping, manufacturing, and market launch (Stevens, 2014). Research has shown that describing and managing a front-end process is difficult and most companies have potential for improvement as it is more complex and harder to describe a good front-end of innovation and consequently implement it compared to the later stage-gate process (Gassmann and Schweitzer, 2014). FFE and NPD are often mainly discussed in relation to large companies, but the problems of seeking an idea such as the organization of the new product development process are also critical for start-ups. They are forced to find profitable products that include, but not limited to a product-market-fit, an invention, business model, and a commercialization (Moogk, 2012; Sońta-Drakaczkowska, 2019). Larger companies have a solid idea-to-launch process, but there is a shortage of strong, high-value ideas (Cooper, 2011). In contrast, start-ups have an idea but lack the execution (Moogk, 2012). It is risky for start-ups to develop a full product before testing a concept in the market. For this reason they need to conduct experiments to reveal (i) validity or (ii) invalidity of the original idea, with the latter case changing the idea needs might change (Moogk, 2012). To increase the quality of the outcome from FFE, the need for specific milestones is proposed to manage activities and resources (Shaytan and Laptev, 2015). The development of new products and especially the persistence through the FFE is essential for start-ups and product success.

In recent years, academic formats such as Think.Make.Start. (TMS) from TU Munich emerged, where teams of students are introduced to entrepreneurship in the context of new product development (Martins Pacheco et al., 2020). Some start-ups have emerged in the last 5 years, where the idea originated in the course $(\sim 30)$. The number of teams that have succeeded in establishing themselves as a start-up is low $(\sim 7)$. There seems to be no scarcity of ideas, but there is a lack of transforming ideas into tangible and desirable products. Initially, the methodology TAF: The Agile Framework was used in the course to transform ideas into prototypes (Hostettler et al., 2017). Elements such as the three lenses of human-centered design (HCD) help to test ideas and evaluate their potential, are combined with PDCA cycles to guide the teams (Deming, 1989; IDEO, 2015). In addition, the lenses of HCD serve as metrics for successful products as they are only successful when addressing all lenses. TAF was used for 4 years in the course but posed the problem that the methodology was unclear due to lack of goals and its effectiveness could not be sufficiently demonstrated, while being focussed exclusively on academic use cases. Consequently, the methodology role-based prototyping (RBPA) was introduced for both fuzzy front-end and industry scenarios to lead interdisciplinary teams from an idea to a product (Martins Pacheco et al., 2020, 2021). The methodology combines elements from TAF (Hostettler et al., 2017), Prototyping For X (Menold et al., 2017), Design Thinking and Scrum (Vetterli et al., 2013) and offers a roadmap with prototype milestones (What to do?) and role concept 
(Who best to do it?). First applications in industry and academia innovation projects are promising but need further testing and specification.

This is where we want to provide a roadmap by proposing a problem focussed and formalized prototyping approach for FFE engineering linked to a role-based model to support start-up teams by showing its application in a case study. The following research questions are addressed: (RQ1) How can start-ups with little to no systems design experience, with just an idea for a mechatronic product, navigate fuzzy front-end product development? and (RQ2) Are there clear role-dominant phases in the development of NPD for start-ups/fuzzy front-end products?

In section 2 of this paper, the development of the framework for start-ups is outlined, followed by its first deployment. In section 3 the results from the deployment are presented and followed by the discussion in section 4 and the conclusion in section 5.

\section{METHODOLOGY}

This study uses Angsa Robotics as a test setting for data collection. Angsa is an early-stage start-up focussing on the development of an autonomous trash picking robot. The goal is to develop a minimum viable product for pilot projects. To achieve this goal the developed framework PETRA is used. This mixed methods research contains two distinct activities for data collection: (1) measurements of prototyping process during the development, and (2) data analysis.

\subsection{Research Approach}

In this engineering-related research, Design Science Research (DSR) is used as a research approach (Hevner et al., 2004). With the application of DSR, this research aims at the creation of an artifact in the form of a framework as it allows for flexibility in the design process of the artifact. The approach is divided into three cycles: (1) Relevance-, (2) Design-, and (3) Rigor Cycle. The relevance cycle is represented by early-stage start-ups with just an idea as the platform for which a framework is designed to support them in early development stages. The environment creates challenges during this research as numerous stakeholders need to be considered along the process. Their insights and influence on the framework from experiments are integrated via feedback-loops. The best practice for new product development, prototyping, and start-ups, as well as opportunities for designing the framework are considered. The knowledge base in this research is represented by state-of-the-art literature for innovation, new product development, fuzzy front-end, prototyping, and further design methods. The created artifact is the Plan-Execute-Test-Reflect-Assimilate (PETRA) framework developed in this research and it incorporates previous artifacts. For creating the framework, the environment with its stakeholders and requirements, and the knowledge base is considered. An overview of how DSR was used in this research is given here (see Figure 1):

1. Research potential was defined by observing the environment and the domain for the framework during entrepreneurship courses (e.g. TMS) and innovation projects with industry.

2. Basic review of literature on ways of teaching applied innovation, entrepreneurial education, prototyping, design thinking, agile, lean, and systems design was conducted.

3. Stakeholder interest was identified.

4. Important aspects and insights on prototyping and new product development form the knowledge base and environment.

5. Intensive review literature was conducted on prototyping, new product development, fuzzy frontend, innovation, systems design, and interdisciplinarity.

6. From the data collected, interdisciplinary teams in new product development environments were identified as a research unit for the framework.

7. Analysis of the current prototyping processes of participants in TMS and mechatronic product start-ups was conducted.

8. Based on previous insights the macro and micro process for the methodology of interdisciplinary teams for new product entrepreneurship courses as TMS was defined.

9. Best practice of the applied methodology in TMS were understood in detail.

10. Gathered insights on the used methods and the knowledge from the analysed literature were used to define framework further for interdisciplinary teams and add tools to it. 
11. Evaluation was conducted as a case-study at Angsa Robotics.

12. Software tool for validation incorporating the framework is in development.

In this research we are only addressing points 10 and 11 . The rest of the points are out of scope.

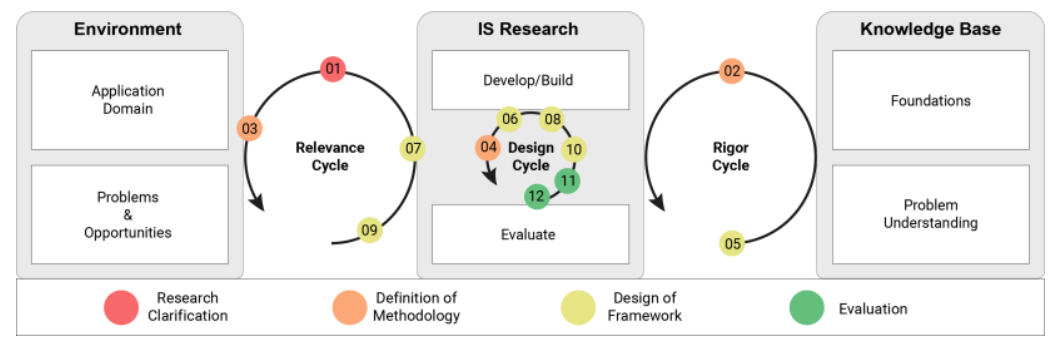

Figure 1. Practical approach of this research according to DSR (Hevner et al., 2004).

\subsection{PETRA - A Fuzzy Front-End Product Development Framework for Start-ups}

The development of new products starts with an idea or a concept that leads to varied, interconnected complexities on the individual, firm, network, and industry level (Ardito et al., 2015). This research tries to address all level as the increase of complexity in product development processes leads to uncertainty and a variety of decisions that influence the early phases. Those early phases are called fuzzy front-end (FFE). To facilitate FFE development, we propose a framework that emphasizes the problem formulation as a first direction for the further development, followed by the development of a minimum viable product (MVP) that focuses on a user-centred design approach. PETRA (PlanExecute-Test-Reflect-Assimilate) is a unified role-based framework that supports new product development by navigating from macro-scaled, requirements-based functionalities development to micro-scaled features development. PETRA builds on existing research on the adapted role-based prototyping approach (Martins Pacheco et al., 2020, 2021). Thereby, prototyping describes the process of creating representations of critical elements of the design and testing hypothesis (Lauff et al., 2017). In addition, the concept of pretotyping preceding prototyping has been added, which represents a simplified experimental model (Savoia, 2019). The FFE development is divided into three metaphases (planning-, concept-, and launch phase) on the macro level to create an adapted double diamond model (see Figure 2) (Ball, 2019; Ulrich and Eppinger, 2011).

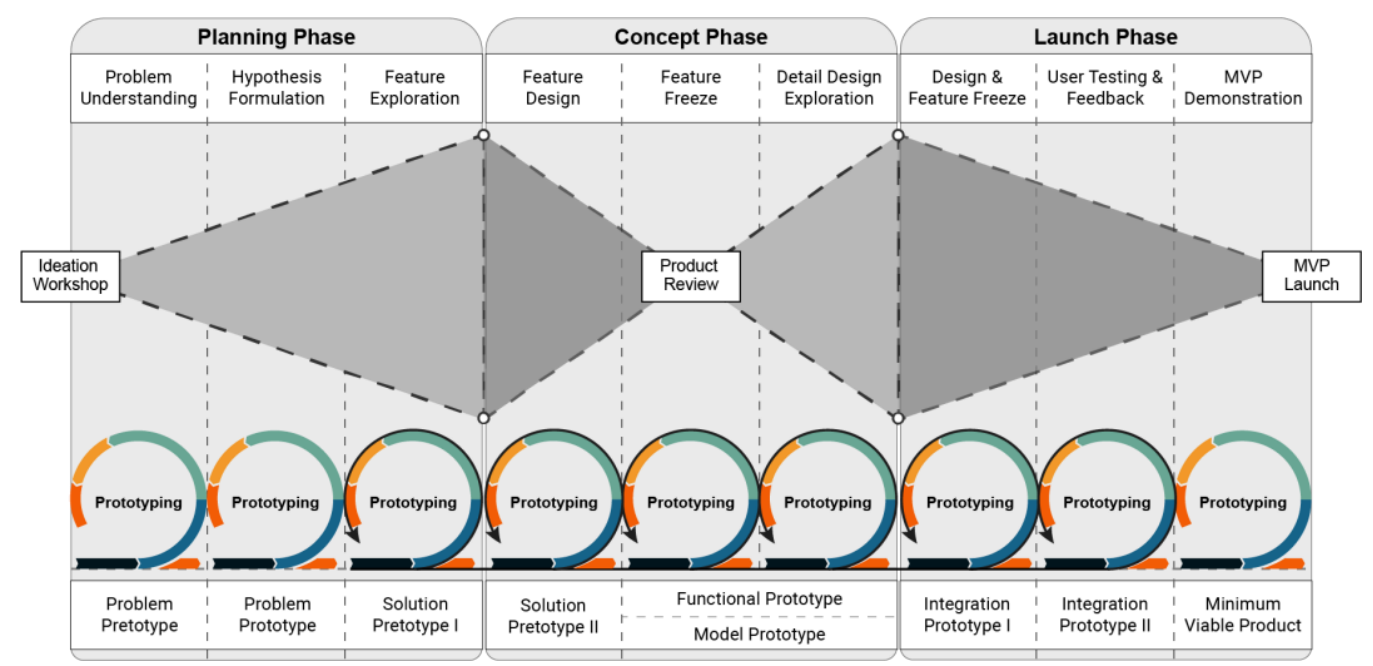

Figure 2. PETRA double diamond model with metaphases for fuzzy front-end development.

The metaphases are divided into nine prototype milestone phases. The problem pretotype (P1) focuses on understanding the problem. The problem prototype (P2) aims at defining the final set of problems that can be reasonably deduced to be solved through targeted user feedback. The solution pretotype (S) is a partially mocked-up representation of the intended product or service that can be built in minutes, hours, or days instead of weeks, months, or years. The functional prototype $(\mathrm{F})$ is a works-like version of the solution with a minimal set of functionalities deployed on the product. The model prototype $(\mathrm{M})$ is a looks-like representation of the product without functional features deployed, but in a presentable form 
for purposes such as user interaction and marketing. The integration prototype (I) combines the workslike and looks-like of the solution. The minimum viable product (MVP) combines both the model- and functional prototypes and contains sufficient features to satisfy early adopters and convince them to invest in the product. These phases are not rigidly sequential but acts as an iterative model that allows for agility, and its major purpose is to serve as a roadmap along the development process.

On a micro level, a typical prototyping cycle is developed with different ceremonies and tools (see Figure 3). A prototyping cycle typically takes two weeks of time and comprises preparation, kick-off, development, review, and documentation of the cycle. In the beginning, an ideation workshop helps to create a vision and develop the product's USP by identifying key markets, core functionalities and usermarkets. In the kick-off, core functionalities to be developed are identified, epics defined and assigned to the respective roles. During the development, everyone works simultaneously in their given roles. After the cycle completion, a reflection session takes place where learnings, shortcomings, and concrete next steps are defined and documented. This is the start to populate the backlog for the next cycle.

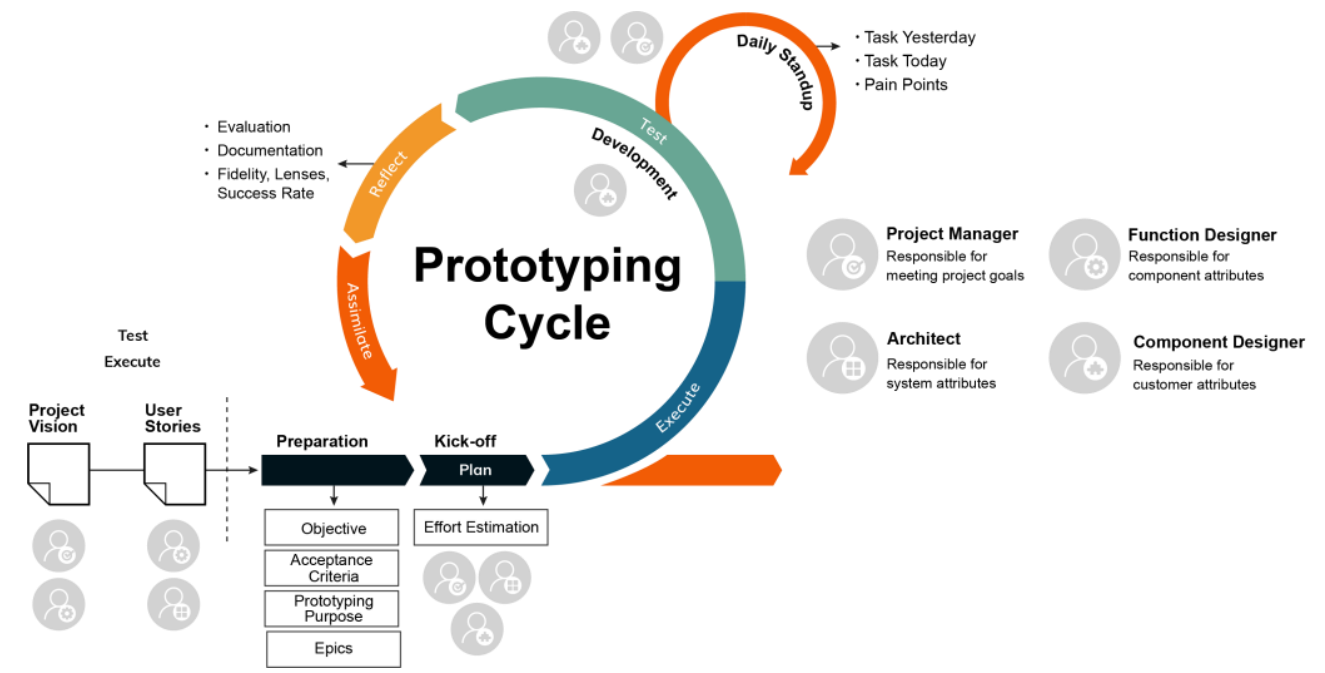

Figure 3. The typical prototyping cycle including roles and ceremonies.

The central tool to document information about each cycles' progress is a modified Kanban board: the "Protoban-Board". The detailed design of this tool is prototyping-focussed and is beyond the scope of this paper. Task cards can be added and are assigned to the status: backlog, work in progress, or completed. A task includes an effort from a Fibonacci scale between 1 (low) and 13 (high) and is rated through the team at each cycle kick-off by the extent of the task. In addition, the objective, and the acceptance criteria (Definition of Done) for the cycle are communicated and visualized on the board. The prototyping purposes: exploration, evaluation, and communication (Blomkvist and Holmlid, 2011); and the chosen prototype milestone phase (see Figure 2) are documented in the cycle kick-off. In the cycle review, the outcome, testing methods, and key insights are documented. The prototyping cycle is rated on a scale between 1 (low) and 5 (high) by the team, while referring on how well the objectives were achieved. In addition, the cycle outcome is assigned to the lenses of HCD: desirability, feasibility, and/or viability (IDEO, 2015). To document the team and work effort, the effort points from the tasks are summed up for each team member. On a team level, clear responsibilities, task allocation, and functions for each team member are provided through predefined roles: Project Manager (PM), Function Designer (FD), Architect (Ar), and Component Designer (CD) that are based on previous research (Martins Pacheco et al., 2021).

The teams are guided through the framework by using prototyping cycles. These cycles collect data at every step on the lenses of human-centred design, the prototyping purposes, and then evaluated by a cycle rating through the entire team during the reflect step. This data is used to summarize progress by means of a prototyping matrix. This matrix highlights the areas that were already considered and highlights missing areas of development and guides on what could be done next without enforcement. The purposes need to progress holistically across the metaphases, i.e., there needs to be a completion of 
all tasks related to all prototyping purposes in each metaphase before it can move to the next metaphase. This allows the team to have a holistic view on the stage of their development in the overall process.

\subsection{Case Study: Angsa Robotics}

The first deployment of the PETRA framework was conducted at Angsa Robotics. The roadmap of the PETRA framework comprising the prototyping milestone phases was used to iteratively plan the development. The first two prototype milestones were covered beforehand in cycle 0 (not part of the case study) and thus recapped in the ideation workshop. The development started with the solution pretotype as the first milestone. The proposed team roles were assigned to team members at Angsa, comprising: one project manager, two architects, one function designer, and two component designers. The two Ar and FD worked also partly as CD. Each CD was responsible for their own component. As a ceremony, daily stand-ups were encouraged to promote interdisciplinary communication. The prototyping cycle was set to three weeks (due to limitations of remote working while COVID-19 restrictions), each cycle started with a cycle preparation and a kick-off. Cycle epics were defined in the preparation and evaluated for effort and assigned to respective roles in the kick-off. After the development phase, the cycle was reflected in the review and documented. The reflection of the cycle was used for the preparation of the following cycle. Everything from the project was tracked with the "Protoban-Board". A digital version of it was used for task tracking for ease of execution (project management software "Trello"). In addition to the proposed status, two more status were added: blocked and long-term backlog. The objective, acceptance criteria, and prototyping purposes were communicated during the kick-off of each cycle and documented visually on "Trello". The tasks were then rated with the entire development team with the planning poker methodology (Cohn, 2005). In the cycle review, each team member presented their prototypes along with key insights and next steps. The completed effort points were summarized and documented for each team member. The success rate of the cycle was rated on a discrete scale between 1 (low) and 5 (high). The prototypes were documented according to the HCD lenses. After the review, all parameters were documented to allow analyses throughout the cycles.

\section{RESULTS}

In this section, the results of applying the PETRA framework in the Angsa project are shown.

\subsection{Success rate and Fidelity across the cycles}

The progress of the success rate $S_{i}$ and fidelity $\mathrm{F}$ over the cycles are shown in Figure 4. $S_{i}$ is closely linked to the prototyping purpose and the fidelity is linked to the overall objectives of the product functionality. The calculation of the success rate for a respective cycle $i$ is done with equation (1).

$$
S_{i}=\frac{1}{11}\left(6 C_{i}+20 \frac{E_{d, i}}{E_{a, i}}+5\right)
$$

$S_{i}$ is the calculated success rate of cycle $i . C_{i}$ represents the rating by the team after a cycle is concluded. $E_{d, i}$ are the efforts that were concluded in the respective cycle $i . E_{a, i}$ are the all the efforts that were defined in the respective cycle $i$.

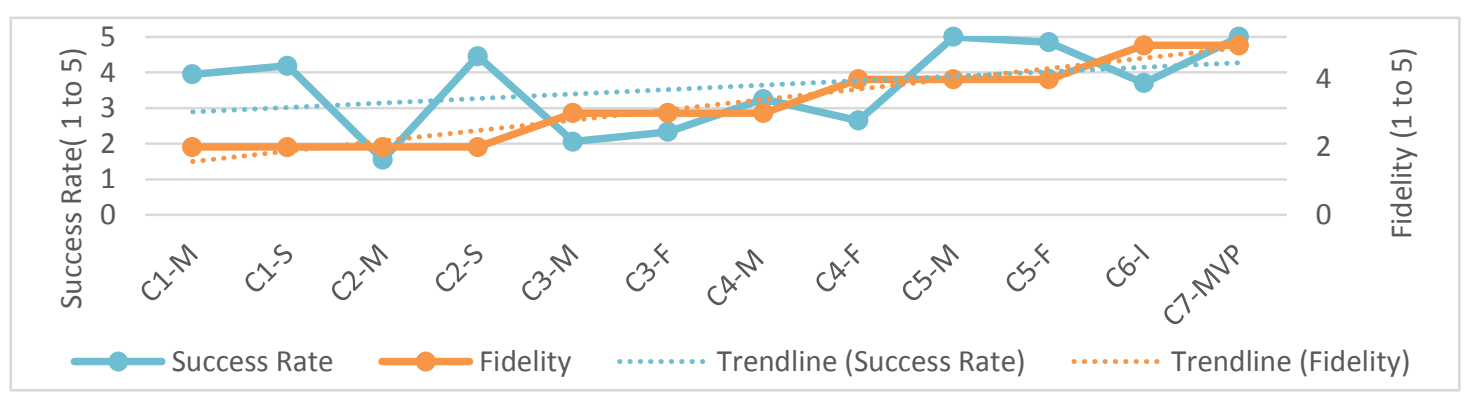

Figure 4. Fidelity and success rate development over the cycles. The average success rate was 3,5. Both metrics have an increasing linear trend towards the end.

It can be observed that the prototyping approach was extremely important in the solution pretotyping and functional prototyping phases, where it alternates between low and high success between the cycles. It can be attributed to better product understanding through solution exploration across cycles. 
The results show that the product fidelity steadily increases across all cycles and that the epics were closed in an incremental fashion by periodic release of prototypes with complete functionalities. All major epics, and core functionalities were realized at the end of the development process successfully.

\subsection{Effort of roles across cycles}

An overview of team roles' effort points in each cycle can be obtained from Figure 5. It needs to be noted that there were more component designers (CD), than architects (Ar) and function designers (FD). The project manager (PM) role was entirely left out since the major work done by the PM was coordinating the team and keeping an overview of the entire process, while his measurable tasks were mostly in the first metaphase. It can be observed that in the initial phases, the roles were going through a divergent phase where the solution exploration led to lower cumulative efforts in all roles. This is evident in the effort points left in work in progress and backlog, while there is an accumulation of completed tasks in the convergent phase. It shows that the FD played a bigger role in the earlier solution prototyping cycles. The Ar focussed mostly on the model prototypes after the initial stages, while the CD focussed mainly on solution and functional prototyping cycles. All three roles converged towards the end, with most of the tasks being completed by $\mathrm{CD}$ for the cycles at the end of the double diamond.

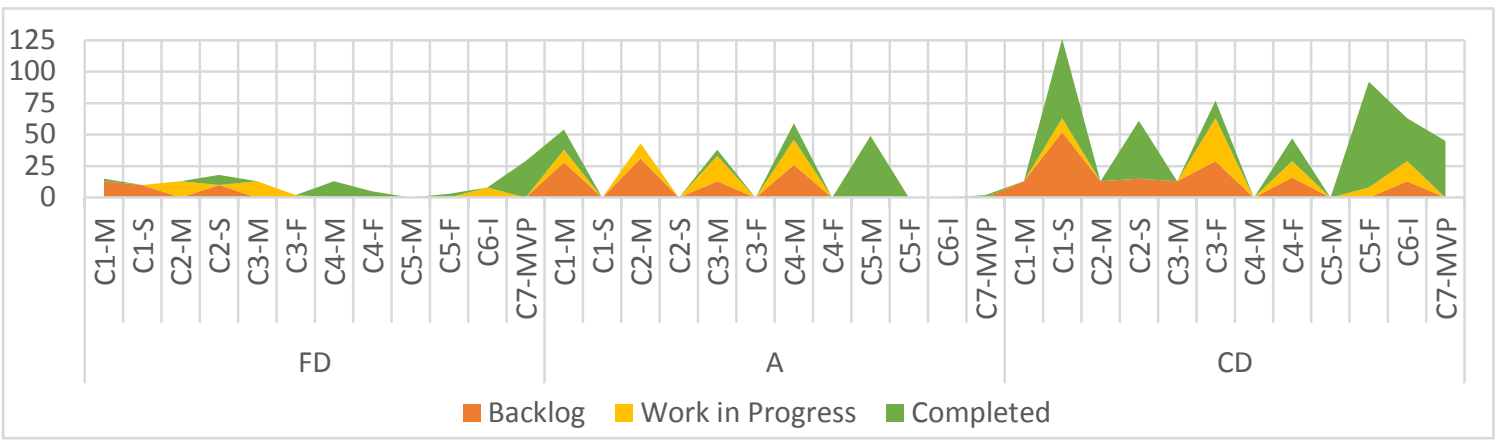

Figure 5. Effort points by cycle and team role (without PM).

\subsection{The matrix of development through the prototyping journey}

The prototyping purposes and lenses of HCD are clear indicators for product fidelity and an unambiguous metric for product development. By combining the lenses and purposes, we derive a development matrix that provides a singular "holistic-progression" indicator matrix. This acts as a compass for the entire development process and gives an overview on where the product stands on its journey to MVP. The columns indicate the lens, and the rows indicate the purpose, and a value formulation is done as shown in equation (2).

$$
\boldsymbol{M}_{i}=\frac{s_{i}}{5 \boldsymbol{l}_{i}^{T} \mathrm{e}} \boldsymbol{p}_{i} \cdot \boldsymbol{l}_{i}^{T} \quad, \boldsymbol{l} \in\{0,1\}^{3} \quad, \mathbf{p} \in(0,1)^{3}
$$

$M_{i}$ is the matrix of cycle $i$. The aggregation of different $M_{i}$ from previous cycles acts as the measure of progress. $S_{i}$ represents the calculated success rate for the considered cycle $i$ (see section 3.1). The vector $l$ represents whether a lens (desirability, feasibility, and/or viability) has been addressed (true $=1$, false $=0$ ). The vector $e$ is the eigenvector with the length one. The vector $p$ represents the purpose in percent that is fulfilled in cycle $i$. This is closely related to the epics and functionalities that were initially drawn to define when the MVP is done, and the rate of success across each cycle. The matrix of development for the Angsa project is shown in Figure 6.

\section{DISCUSSION}

From using the proposed PETRA framework, we identified weaknesses and strengths by collecting data on its applicability and utility. Here, we discuss how this data answers each of the posed research questions.

\subsection{Guiding the Fuzzy Front-End Product Development}

The collected data shows the progression of the development while looking at the matrix of development. We found that specific milestones tended to address particular lenses and purposes. It 
can be observed how the progression changed according to the priorities placed at every cycle. We can also observe how most activities were done through the feasibility lens for the functionality focussed prototypes, while desirability was the focus for the model prototypes, and viability had equal importance across purposes. We also observed at cycle 6 , there was a need for an integration phase when both functionality and the model prototype had to merge before the MVP design was complete. This is an extended prototyping cycle towards MVP. Since it had its own development purpose and lens associated with it, we decided to treat it like a separate prototype, that merged all functionalities. However, the effectiveness of the matrix is dependent on the carefully planned definition of done and product epics. The holistic progression in the product development process is achieved by keeping an eye on all key functionalities. For this, the scores for the prototyping purposes are limited at every metaphase. Individual prototyping cycles with lower success scores maintained a holistic view in the double diamond method and kept the success rate linearly progressive. By making the development process measurable, we can show what has been done and give better guidance on what to do next. We think that there is value by measuring the development process, as for instance it indicates the position of a development team in the development process and shows the current fidelity of the product.

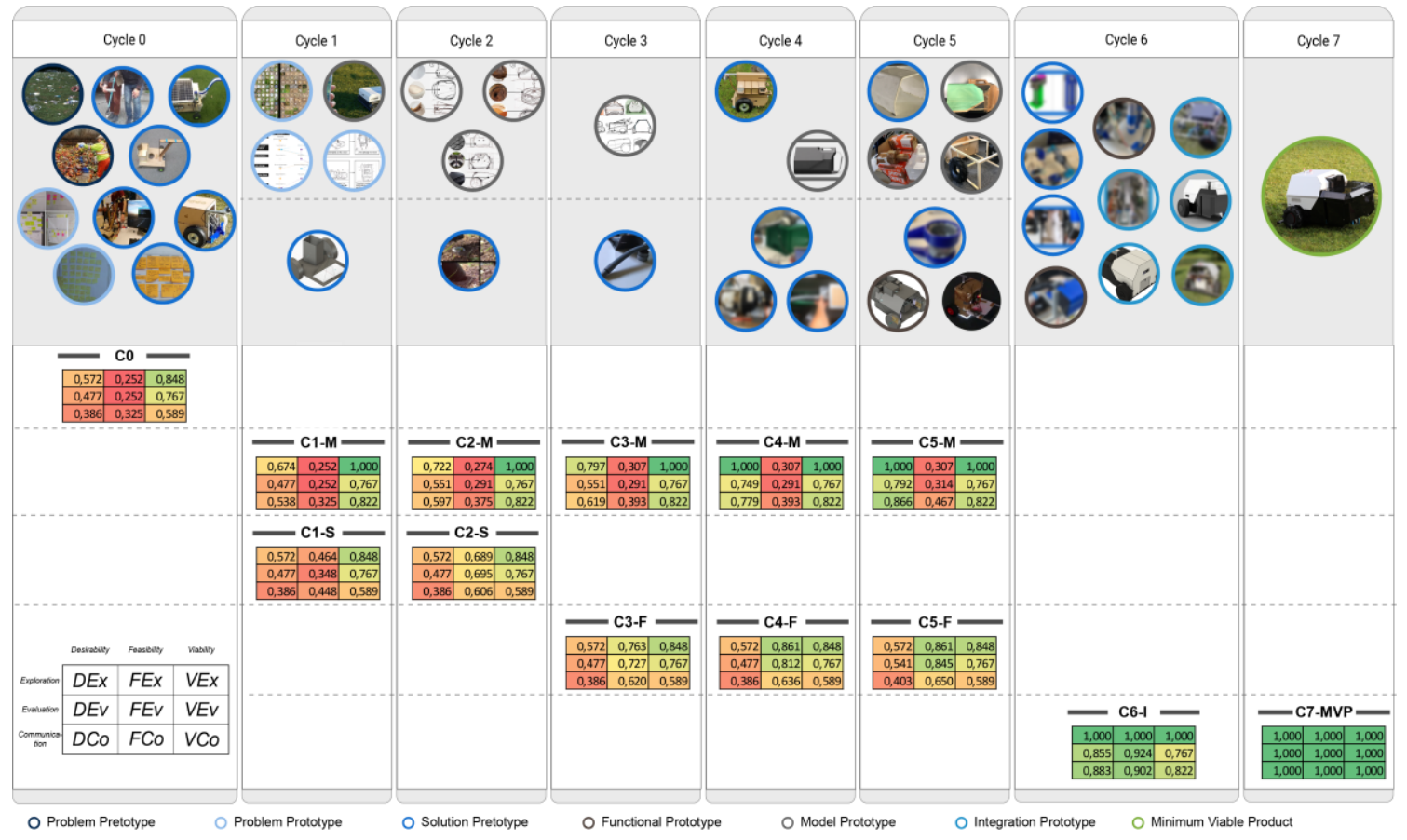

Figure 6. Angsa prototyping journey and the matrix of development. It can be observed that the functionality-oriented cycles progressed along the feasibility lens and the prototypes built for interaction design and commercial viability was mostly oriented towards the desirability lens. The prototypes shown are the results after a prototyping cycle. Cycle 0 is excluded from the consideration (*blurred pictures as disclosure of technical details not possible)

\subsection{Role-dominant phases in the development of new products}

A measurement of team role efforts in the data collection allowed us to identify areas of prototyping where specific roles were more engaged. In the divergent phase, most of the effort comes from the architect (Ar) and the function designer (FD), while the convergent phases relate to the component designer (CD). This way, the role understanding helped to specify responsibilities and contributed to the allocation of personnel resources more efficiently. This study compared the amount of effort between different roles in the development process. The data did not conclude that the FD, Ar, and CD did most of the work compared to the project manager (PM). The effort was only measured in the beginning of a cycle (defined tasks) and at the end of a cycle (concluded tasks). This approach did not capture all tasks that had to be done immediately by the PM, since creating already completed tasks afterwards did not serve the development progress. These efforts were not considered in the data collection as no corresponding tasks were created in the Protoban-Board. Therefore, the role of the PM needs to be excluded in this study. In addition, we see that in the beginning a lot of tasks were defined that needed to be done in the future. Most of the tasks went directly into the backlog, and only towards 
the end, more tasks were concluded. The reason is straightforward, there are tasks which have a dependency from other tasks and tasks that are independent. The number of tasks closed during the makeathon phase did not vastly increase as compared to the prototyping cycle, this could be due to clear planning of milestones that kept the completion of functionalities consistent. We recommend adding the possibility to document all tasks that were done by giving an effort to the task, even if it is a small task. Measuring the effort of different roles in more studies has the potential to provide better insights on what was done by whom. We think that those insights help to better allocate personnel resources in the different stages of the development project.

\subsection{Limitations and Outlook}

In this study, we examined the data collected in the development project at Angsa, but the question remains whether the proposed framework is generally applicable to different start-ups with other scenarios. While the framework with all its details was explained to the development team, a risk remains that success of the development was influenced by other factors such as the feeling of progression in the project not measured in this study. To ensure reliability and validity this research refers to Roberts et al. (2006). The reliability of this research is addressed by providing templates to be filled out so that data can be easily digitized and coded to conduct data analysis with different statistical programs. To overcome any researcher bias in the interpretation of data, different researchers audited the results from the analysis. The validity of this qualitative research is addressed by giving the team a framework incorporating different tools to use the methodology accordingly. Different templates help the development team to use the framework without prior experience. During the project, a research diary was created to document all steps in the research. This paper is a beginning to make early development processes with respect to prototyping measurable. The results are a good first step to prove the effectiveness of the framework. Compared to studies on role-based prototyping (Martins Pacheco et al., 2020, 2021) and evaluation studies on TAF - The agile Framework (Spreiter et al., 2018), this study enabled to collect consistent data on the product development process over a period of 5 months with not only describing it, but also making it measurable by using the PETRA framework. Currently, this framework is designed for start-ups developing a mechatronic product, but the application can be extended to innovation projects in any kind of scenario that want to quickly test new ideas and address the fuzzy front-end development for products or processes, but the proposed variation and a corresponding tool incorporating the framework is being currently developed.

\section{CONCLUSION}

This paper contributes to the question on how to guide start-ups through the fuzzy front-end development. Specifically, this study investigated the applicability and usability of the PETRA framework in a case study with a start-up developing an autonomous trash picking robot. We extended the role-based prototyping approach by adding a prototyping matrix with two dimensions (purpose and lens), a particular prototyping cycle (plan, execute, test, reflect, assimilate), and a modified Kanban board (Protoban) for planning, managing, and reflecting cycles. This provides the tools, a way to allocate resources efficiently, and a procedure to prototype iteratively towards the minimum viable product. This way, the development process becomes more focused and efficient while providing sufficient freedom in early phase design work. It supports the development team significantly in providing a clear idea of what to do at what instance with respect to (1) the prototyping process, (2) team organization and dynamics, and (3) progress tracking. It is mainly based on the qualitative and quantitative assessment of the development results. The results show that the PETRA framework has successfully supported the development project and that specified roles helped to allocate personnel resources more efficiently.

\section{ACKNOWLEDGMENTS}

The authors would like to thank the Angsa Robotics team for their kind support and collaborative input for this research.

\section{REFERENCES}

Ardito, L., Messeni Petruzzelli, A. and Albino, V. (2015), "From technological inventions to new products: A systematic review and research agenda of the main enabling factors", European Management Review, Wiley Online Library, Vol. 12 No. 3, pp. 113-147. 
Ball, J. (2019), "The double diamond: A universally accepted depiction of the design process", The Design Council UK Https://Www.Designcouncil.Org.Uk/News-Opinion/Double-Diamond-Universally-AcceptedDepiction-Design-Process.

Blomkvist, J. and Holmlid, S. (2011), "Existing prototyping perspectives: considerations for service design", Nordes, No. 4.

Cohn, M. (2005), Agile Estimating and Planning, Pearson Education.

Cooper, R.G. (2011), Winning at New Products: Creating Value through Innovation, Basic Books.

Crawford, C.M. (2008), New Products Management, Tata McGraw-Hill Education.

Deming, W.E. (1989), “Out of the Crisis. Quality, Productivity and Competitive Position”, Massachusetts Institute of Technology, Cambridge, MA, Vol. 81, p. 82.

Florén, H., Frishammar, J., Parida, V. and Wincent, J. (2018), "Critical success factors in early new product development: a review and a conceptual model”, International Entrepreneurship and Management Journal, Springer, Vol. 14 No. 2, pp. 411-427.

Gassmann, O. and Schweitzer, F. (2014), Management of the Fuzzy Front End of Innovation, Springer.

Hevner, A.R., March, S.T., Park, J. and Ram, S. (2004), "Design science in information systems research", MIS Quarterly, JSTOR, pp. 75-105.

Hostettler, R., Bohmer, A.I., Lindemann, U. and Knoll, A. (2017), “TAF agile framework reducing uncertainty within minimum time and resources", 2017 International Conference on Engineering, Technology and Innovation (ICE/ITMC), Vol. 2017, IEEE, pp. 767-775.

IDEO. (2015), The Field Guide to Human-Centered Design, Design Kit.

Kahn, K.B., Castellion, G. and Griffin, A. (2005), The PDMA Handbook of New Product Development, Wiley Online Library.

Karniel, A. and Reich, Y. (2011), Managing the Dynamics of New Product Development Processes: A New Product Lifecycle Management Paradigm, Springer Science \& Business Media.

Koen, P.A., Ajamian, G.M., Boyce, S., Clamen, A., Fisher, E., Fountoulakis, S., Johnson, A., et al. (2002), "Fuzzy front end: effective methods, tools, and techniques", The PDMA Toolbook, Vol. 1, pp. 5-35.

Lauff, C., Kotys-Schwartz, D. and Rentschler, M.E. (2017), "What is a prototype?: Emergent roles of prototypes from empirical work in three diverse companies", International Design Engineering Technical Conferences and Computers and Information in Engineering Conference, Vol. 58219, p. V007T06A033.

Martins Pacheco, N.M., Behrenbeck, J., Tariq, B., Vazhapilli Sureshbabu, A., Zimmermann, M. and others. (2020), "A Role-based Prototyping Approach for Human-Centred Design in Fuzzy Front-End Scenarios", DS 101: Proceedings of NordDesign 2020, Lyngby, Denmark, 12th-14th August 2020, pp. 1-12.

Martins Pacheco, N.M., Vazhapilli Sureshbabu, A. and Zimmermann, M. (2021), “A Role-Based Prototyping Approach for Human-Centered Design in Industry”, ICoRD’21--Design for Tomorrow (Accepted), Springer.

Menold, J., Jablokow, K. and Simpson, T. (2017), "Prototype for X (PFX): A holistic framework for structuring prototyping methods to support engineering design", Design Studies, Vol. 50, pp. 70-112.

Moogk, D.R. (2012), "Minimum viable product and the importance of experimentation in technology startups", Technology Innovation Management Review, Vol. 2 No. 3.

Roberts, P. and Priest, H. (2006), "Reliability and validity in research", Nursing Standard, Royal College of Nursing Publishing Company (RCN), Vol. 20 No. 44, pp. 41-46.

Savoia, A. (2019), The Right It: Why So Many Ideas Fail and How to Make Sure Yours Succeed, HarperCollins.

Shaytan, D.K. and Laptev, G.D. (2015), "Exploring Management of the Fuzzy Front End of Innovation in a Product Driven Startup Company”, International Journal of Economics and Management Engineering, Vol. 9 No. 4, pp. 1303-1308.

Sońta-Drakaczkowska, E. (2019), "New Product Development in High-tech Startups-a Conceptual Framework", Marketing i Rynek, No. 1, pp. 30-37.

Spreiter, L., Böhmer, A.I., Lindemann, U. and others. (2018), "Evaluation of TAF agile framework based on the development of an innovative emergency wearable for seniors", DS 92: Proceedings of the Design 2018 15th International Design Conference, pp. 1345-1356.

Stevens, E. (2014), "Fuzzy front-end learning strategies: Exploration of a high-tech company", Technovation, Elsevier, Vol. 34 No. 8, pp. 431-440.

Von Thienen, J., Noweski, C., Meinel, C. and Rauth, I. (2011), "The co-evolution of theory and practice in design thinking--or-'mind the oddness trap!'”, Design Thinking, Springer, pp. 81-99.

Trott, P. (2016), Innovation Management and New Product Development, Pearson.

Ulrich, K. and Eppinger, S. (2011), Product Design and Development, McGraw-Hill Education.

Vetterli, C., Übernickel, F., Brenner, W., Haeger, F., Kowark, T., Krüger, J., Müller, J., et al. (2013),

"Jumpstarting scrum with design thinking", University of St. Gallen. 